\title{
REVISIONES
}

\section{Educación patrimonial: retrospectiva y prospectivas para la próxima década*}

\author{
Heritage Education: Retrospective and Forecast for the next decade
}

Educação patrimonial: retrospectiva e prospectivas durante década que vem

\author{
Olaia Fontal Merillas
}

Universidad de Valladolid, España

Telf.: (34) 983184676. Correo electrónico: ofontal@mpc.uva.es

\begin{abstract}
El papel de la educación en los procesos de puesta en valor y socialización del patrimonio está siendo internacionalmente reconocido, hasta el punto que algunos países, como es el caso de España, se han dotado en la última década de instrumentos eficaces para la gestión educativa de su legado. Proponemos un recorrido que comienza por definir el estado de la educación patrimonial en el ámbito internacional, acercándonos al caso de Europa y, dentro de este continente, España. Todo ello nos va a permitir conocer algunas características de los programas educativos en materia de patrimonio, para sí poder definir modelos y estándares que guíen las acciones de los diferentes países en educación patrimonial para la próxima década.
\end{abstract}

Palabras clave: educación patrimonial, patrimonio cultural, evaluación de programas, legislación educativa.

\begin{abstract}
The role of education is very important in the processes of appreciation and socialization of heritage. In the last decade, some countries -as Spain- have even created some effective instruments for the educational management of its legacy. We propose an article that starts defining the condition of heritage education in the international scope, approaching to the European case and, inside the continent, the Spanish one. All these will allow the recognition of the characteristics of educational programs regarding heritage to be able to define models and standards that can guide the actions of the different countries in heritage education for the next decade.
\end{abstract}

Key words: heritage education, cultural heritage, evaluation of programs, educational legislation. 


\section{LA IMPORTANCIA DE LA EDUCACIÓN EN LAS CONVENCIONES INTERNACIONALES DE PATRIMONIO}

En la última década, la literatura científica sobre patrimonio —especialmente la procedente de las disciplinas de la educación, comunicación y turismo- ha contribuido a superar la visión objetual del patrimonio (el patrimonio son bienes, cosas, elementos tangibles); la visión legislativa (el patrimonio es aquello que se declara como tal en la legislación internacional, estatal y autonómica); la visión monumentalista (el patrimonio se refiere a bienes de gran presencia y monumentalidad); la visión historicista (el patrimonio ha de tener tiempo, peso y poso de la historia); la visión economicista (el patrimonio comprende preferentemente bienes con un elevado valor económico); la visión universalista (el patrimonio puede llegar a pertenecer al conjunto de la humanidad, como es el caso de la categoría de patrimonio mundial de la UNESCO); y la visión turístico-lúdica (el patrimonio ha de estar vinculado a espacios, lugares, sitios o espectáculos que desean ser visitados por las personas, especialmente por aquellas ajenas al contexto en el que se inscribe).

Todos estos enfoques, si bien siguen siendo una parte clave en la concepción del patrimonio en tanto conforman su esencia, resultan incompletos o han ido incorporando otras visiones más holísticas (Cuenca, 2002, 2003; Cuenca y Estepa, 2013; Estepa, 2013;) gracias, en gran medida, al proceso de teorización generado desde la Didáctica de las Ciencias Sociales y, en menor medida, desde la Educación Artística. En primer lugar, una visión personalista que da importancia al sujeto en tanto agente activo en la conformación del patrimonio (Fontal, 2003, 2013); es el ser humano quien crea los bienes patrimoniales, los custodia, investiga, valora y transmite. Se incorpora, también, la dimensión identitaria o simbólico-social que sitúa el acento en la capacidad de simbolizar y en el potencial para generar procesos de identización (Gómez Redondo, 2011, 2012) que confiere un gran "poder" a los bienes patrimoniales; una cuestión derivada de esta es la de los posibles usos que se puedan hacer tomando en consideración ese "poder" identitario, de referenciación, de identificación y de definición que confiere el patrimonio (Ballart, 1997, 2012; Ballart y Tresserras, 2001). También se incorpora la visión procesual, en tanto el legado patrimonial es un conjunto de hilos que cada generación va heredando de las anteriores, tomando irremediablemente decisiones de conservación (ninguna generación mantiene todo el patrimonio que recibe), de puesta en valor, de recuperación y, además, cada generación debe sumar su propio legado en tanto que el patrimonio es un proceso vivo, en constante transformación (Fontal, 2012). Esto abre las puertas al patrimonio contemporáneo y supera la visión historicista del paso y poso del tiempo, en favor del potencial de identificación y de definición de un contexto social, político, geográfico y cultural. Todas estas visiones suponen una valoración y conceptualización del patrimonio desde la educación e incorporan características del patrimonio cultural considerado potencialmente más próximo a las personas.

Justamente, incorporando todas estas perspectivas más dinámicas, la UNESCO lleva una década redefiniendo su propio concepto de patrimonio. En 2003, año de la primera Convención internacional para la salvaguardia del Patrimonio Cultural Inmaterial, encontramos una definición relativa este patrimonio que bien podría hacerse extensiva a todos los demás patrimonios, desde el histórico, hasta el medioambiental, industrial, etnográfico o arqueológico, en tanto incorpora los elementos "esenciales" del sustantivo patrimonio y del adjetivo patrimonial (Fontal, Darrás y Rickenmann, 2008). 
Se entiende por 'patrimonio cultural inmaterial' los usos, representaciones, expresiones, conocimientos y técnicas -junto con los instrumentos, objetos, artefactos y espacios culturales que les son inherentes- que las comunidades, los grupos y en algunos casos los individuos reconozcan como parte integrante de su patrimonio cultural. Este patrimonio cultural inmaterial, que se transmite de generación en generación, es recreado constantemente por las comunidades y grupos en función de su entorno, su interacción con la naturaleza y su historia, infundiéndoles un sentimiento de identidad y continuidad y contribuyendo así a promover el respeto de la diversidad cultural y la creatividad humana. A los efectos de la presente Convención, se tendrá en cuenta únicamente el patrimonio cultural inmaterial que sea compatible con los instrumentos internacionales de derechos humanos existentes y con los imperativos de respeto mutuo entre comunidades, grupos e individuos y de desarrollo sostenible (UNESCO, 2003).

Esta definición destaca las dimensiones inmaterial y espiritual del patrimonio que, si bien habían estado presentes en las propias definiciones de UNESCO ya desde 1972 en la Convención sobre la protección del patrimonio mundial, cultural y natural, toman ahora un papel protagónico, casi esencial. Sin embargo, la importancia de la educación patrimonial ha estado muy presente en todas las convenciones de UNESCO, ya desde 1972, cuando se incluía un capítulo con dos artículos dedicados explícitamente a la educación en la Convención sobre la protección del patrimonio mundial, cultural y natural.

\section{Artículo 27}

Los Estados Partes en la presente Convención, por todos los medios apropiados, y sobre todo mediante programas de educación y de información, harán todo lo posible por estimular en sus pueblos el respeto y el aprecio del patrimonio cultural y natural definido en los artículos 1 y 2 de la presente Convención.

Artículo 28

Los Estados Partes en la presente Convención, que reciban en virtud de ella, una asistencia internacional tomarán las medidas necesarias para hacer que se conozca la importancia de los bienes que hayan sido objeto de asistencia y el papel que ésta haya desempeñado (UNESCO, 1972).

En 2005, la Convención sobre la protección y promoción de la diversidad de las expresiones culturales de UNESCO, ya hace referencia explícita a los procesos de sensibilización, que intenta ordenar, reafirmando el papel fundamental que desempeña la educación en la protección y promoción de las expresiones culturales:

Artículo 10 - Educación y sensibilización del público

Las Partes deberán:

(a) propiciar y promover el entendimiento de la importancia que revisten la protección y fomento de la diversidad de las expresiones culturales mediante, entre otros medios, programas de educación y mayor sensibilización del público (...)

(c) esforzarse por alentar la creatividad y fortalecer las capacidades de producción mediante el establecimiento de programas de educación, formación e intercambios en el ámbito de las industrias culturales. Estas medidas deberán aplicarse de manera que no tengan repercusiones negativas en las formas tradicionales de producción (UNESCO, 2005). 
En síntesis, toda definición de patrimonio derivada de las convenciones internacionales, que tiene en 2003 un punto de inflexión con el revulsivo que supone la dimensión inmaterial del patrimonio, asume que este comprende bienes (materiales, inmateriales y espirituales) a los que las personas atribuyen distintos valores, que además van cambiando en el transcurso de la historia. Por tanto, el patrimonio por sí mismo no tiene valor ni deja de tenerlo; es justamente el ser humano quien se lo confiere y, por ello, los valores son múltiples, cambiantes y educables. Ahí es donde cobra su verdadero valor la acción educativa (Fontal, 2013: 10-12).

\section{PRINCIPALES AGENTES PARA LA EDUCACIÓN PATRIMONIAL EN EL ÁMBITO INTERNACIONAL}

La UNESCO es, sin lugar a dudas, la institución de mayor peso, recursos e implicación en el ámbito del patrimonio a nivel mundial. La UNESCO, entidad dependiente de la ONU, se desarrolla a través de diferentes organismos (ICOM, ERC, etc.), comités, comisiones, consejos, cátedras, universidades, redes y socios. Cuenta con 192 estados miembros con otras tantas comisiones nacionales, de los cuales 180 tienen delegaciones permanentes. En sus secciones de Educación y de Cultura, la UNESCO lleva a cabo acciones en todo el mundo, distribuidas en cinco zonas: a) África, b) Estados Árabes, c) Asia y el Pacífico, d) Europa y América del Norte y e) América Latina y el Caribe. Como muestra de algunas de estas líneas prioritarias, comentamos algunas propuestas generadas desde la UNESCO con vocación internacional. Destacamos algunas redes vinculadas directamente con la educación, formación y difusión del patrimonio.

Red FORUM UNESCO UNIVERSIDAD Y PATRIMONIO. Forum UNESCO-Universidad y Patrimonio (FUUP) es un proyecto de la UNESCO para la realización de actividades para la protección y salvaguarda del patrimonio cultural, a través de una red informal de instituciones de educación superior. FUUP está bajo la responsabilidad común del Centro de Patrimonio Mundial de la UNESCO y la Universidad Politécnica de Valencia (UPV), España. Se creó para apoyar las acciones de la UNESCO en favor de la protección, la mejora y la conservación del patrimonio cultural y natural, entendiendo que las universidades, en tanto que miembros permanentes e independientes de la sociedad civil, educan a las generaciones futuras en los valores de la paz, el diálogo y el respeto entre culturas, de acuerdo con los principios de la UNESCO. Otro objetivo es mantenerse informado o dar amplia difusión a las actividades realizadas por las universidades a través de la base de datos de la red disponible en línea. La Red permite trabajar en equipo con otras universidades en un dominio relacionado con el patrimonio cultural o natural elegido en coordinación con la UNESCO. Gracias a Forum UNESCO, es posible poner en contacto a los profesionales de la conservación del patrimonio con el entorno académico; llevar a cabo intercambios de estudiantes y profesores y compartir programas o actividades (publicaciones, investigación, proyectos, etc.); establecer relaciones entre universidades y otras partes interesadas de la sociedad civil, como por ejemplo medios de comunicación, representantes locales y profesores, para garantizar de forma conjunta la protección del patrimonio; fomentar la creación de programas universitarios y/o cátedras UNITWYN de la UNESCO en las áreas de conservación del patrimonio cultural o natural.

En la Sección UNESCO-CULTURA, destacamos la Red de Ciudades Creativas, que fue lanzada por la UNESCO en 2004, tras el $170^{\circ}$ Consejo Ejecutivo. Su objetivo es fomentar 
el aprovechamiento del potencial creativo, social y económico de las colectividades locales y promover que se persigan los objetivos de la UNESCO respecto a la diversidad cultural. Las ciudades deben informar a la UNESCO cada año acerca del progreso realizado en la implementación de sus políticas y actividades, tanto a nivel nacional como internacional, en cooperación con otras ciudades.

En la Sección UNESCO-EDUCACIÓN destacamos la Red del Plan de Escuelas Asociadas de la UNESCO (redPEA) (en inglés, ASPnet). Se trata de una red mundial que comprende unos 8000 centros escolares en 177 países (escuelas de infantil, primaria y secundaria e institutos de formación superior), en la cual todos trabajan en favor de una educación de calidad. Las escuelas se comprometen a difundir los principios y recomendaciones de la UNESCO, a participar en los proyectos específicos de la UNESCO, destinados a la Red de escuelas asociadas, participar en las reuniones y encuentros, regionales, autonómicos, estatales e internacionales. Deben, también, elaborar cada año una memoria informativa de las actividades y programas realizados como escuela asociada. La pertenencia a la Red es una labor de todo el centro educativo y no de un grupo de profesores. El proyecto RedPEA es coordinado por el World Heritage Centre de UNESCO y el Associated Schools Project Network (ASPnet) financiado por la Agencia noruega para la Cooperación de Desarrollo (NORAD), que anteriormente tuvo el apoyo anterior del Rhône Poulenc la Fundación, Francia. Pretende la integración de la educación patrimonial en el currículum de secundaria y trata de implicar a los jóvenes en la conservación del Patrimonio Mundial. Buscan una sinergia y colaboración entre profesores, responsables en la elaboración del patrimonio, expertos en patrimonio y otra gente relevante en el ámbito local y global. El programa se fundamenta en la creencia de que solo inculcando un sentido profundo de responsabilidad hacia nuestra herencia común entre quienes tendrán el poder de decisión en un futuro, es posible preservar la rica diversidad cultural y natural del planeta.

En el Centro de Patrimonio Mundial de la UNESCO (World Heritage Centre), entre la relación de actividades encontramos la línea de "Education and Training", donde destacan los siguientes programas: Higher education, UNESCO/NHK Videos on Heritage, UNESCOVOCATIONS PATRIMOINE Fellowships for World Heritage Site Managers, World Heritage and the Arctic, World Heritage Education, World Heritage Volunteers, Caribbean Capacity Building Programme (CCBP), France-UNESCO Cooperation Agreement, Handbook on Cultural Heritage y Local Development for African Local Governments Published, Ongoing University Projects supported by UNESCO World Heritage Centre. Destacamos, de entre todos ellos, la propuesta "World Heritage Education", un amplio programa educativo que aporta a la gente joven la oportunidad de involucrarse directa y activamente en la protección del patrimonio cultural y natural del Mundo. Tiene propuestas como el "Kit: World Heritage in Young hands" (Anexo 2), el programa Patrimonito, Recursos, voluntariado de Patrimonio Mundial y otras muchas acciones.

ICOM es una organización profesional de museos orientada a conservar, estudiar y difundir a la sociedad el patrimonio cultural y natural del mundo. Se creó en 1946, es de carácter no gubernamental y tiene un estatus consultivo para el Consejo Económico y Social de Naciones Unidas. Se organiza a través de un conjunto de comités nacionales, internacionales, organizaciones regionales y organizaciones afiliadas. Sus actividades comprenden: Día Internacional del Museo, Conferencias generales, Lucha contra el tráfico ilícito, Formación y reciclaje, Participación de la comunidad, Patrimonio intangible y, finalmente, Gestión de riesgos. Por su parte, $C E C A$ —no de los comités internacionales del 
ICOM más antiguos-, promueve el desarrollo de la educación museal y la acción cultural a través de un sistema de coordinaciones regionales (las cinco regiones de la UNESCO) y, dentro de ellas, publicaciones y un evento anual donde se reflexiona y exponen proyectos singulares y se realiza un diagnóstico del estado de la cuestión internacional.

\section{LA EDUCACIÓN PATRIMONIAL EN EUROPA}

La Unión Europea, a través de la Comisión Europea, desarrolla un amplio programa para la difusión y acceso al patrimonio europeo. Así, dentro de la línea de acción "Preserving and enhancing common heritage" pretenden preservar y poner en valor el patrimonio cultural de Europa, uno de los objetivos prioritarios del programa cultural de la cooperación "Cultura 2000". Financia proyectos para conservar patrimonio europeo de importancia excepcional, denominados "European heritage laboratories".

El Programa Cultura 2000 reúne los antiguos programas "Raphael", "Caleidoscopio" y "Ariane", y tiene por objeto crear un espacio cultural común fomentando el diálogo cultural y el conocimiento de la historia, la creación, la difusión de la cultura de los pueblos europeos y la movilidad de los artistas y sus obras, el patrimonio cultural europeo, las nuevas formas de expresión cultural y el papel socioeconómico de la cultura. "Cultura 2000" apoya proyectos de cooperación transnacionales en los que colaboran creadores, operadores culturales e instituciones culturales de los países que participan en el programa. Se desarrollan, desde este programa, acciones específicas, innovadoras o experimentales en las que intervienen operadores de al menos tres de los países que participen en el programa, acciones integradas en acuerdos de cooperación cultural, estructurados y plurianuales; acontecimientos culturales especiales de proyección europea o internacional. El programa Cultura 2007 sustituye a Cultura 2000.

El Programa Cultura 2000 también proporciona la ayuda para los proyectos de cooperación en el campo del patrimonio como Art Nouveau Network. Abarca proyectos que implican, entre otras cosas, el entrenamiento, el intercambio de experiencias y el desarrollo de productos culturales multimedia. También apoya a "Europa Nostra" con proyectos de restauración del patrimonio.

La preservación del patrimonio es también uno de los objetivos del programa de Tecnologías de la sociedad de información (IST) en el marco de su acción Preservación y desarrollo del patrimonio cultural $\left(6^{\circ}\right.$ Programa de base para la investigación y el desarrollo tecnológico).

Dentro de la línea de acción "Access to common heritage", la Unión Europea desarrolla sus programas de educación y formación y sus campañas de información dentro de Consejo de Europa para promover el acceso público al patrimonio cultural.

El programa de SOCRATES apoya los proyectos educativos en el campo del patrimonio cultural que implican a escuelas y museos; por ejemplo, el programa Leonardo da Vinci, que entre otras cosas financia la formación en artes tradicionales y en la restauración y el desarrollo del patrimonio cultural.

La Unión Europea está implicada en un esfuerzo por aumentar y poner en valor el conocimiento del patrimonio cultural, dentro de un programa denominado "Europe, a common heritage", que fue lanzado en 1999 por el Consejo de Europa. Como parte de esta campaña, la Comisión Europea y el Consejo de Europa han firmado cinco convenciones 
y han lanzado varios proyectos comunes en el marco del programa de la cultura 2000, ocupándose de aspectos como la herencia fotográfica de las universidades antiguas de Europa, la industria de la artesanía y de las tradiciones musicales de Europa.

El Consejo de Europa y la Unión Europea también están trabajando juntos para organizar los Días europeos del Patrimonio (European Heritage Days), que se han llevado a cabo desde 1991. El programa cultura 2000 también financia los proyectos que intentan compartir y poner en valor el conocimiento del patrimonio común europeo.

En el ámbito europeo, destacamos el programa HEREDUC-Heritage Education. HEREDUC es un término compuesto por HERitage y EDUCation. Se trata del primer proyecto que abarca la educación patrimonial a nivel europeo. Forma parte del programa Comenius, dentro del programa Sócrates de la Unión Europea. Participan Bélgica, Alemania, Holanda, Francia e Italia. El proyecto ha desarrollado una guía para profesores de un gran interés (ver Anexo 3). La propuesta se basa en principios como la diversidad entre países, la transferibilidad de principios y modelos de actuación, la interdisciplinariedad cuando se trabaja lo patrimonial, el carácter transversal en el currículo, la conexión con las vidas y vivencias de los alumnos, la cooperación con agentes patrimoniales y la participación activa y determinante del alumnado. Se han analizado hasta la actualidad 34 casos europeos, seleccionados como ejemplos de buenas prácticas que inspiran la creatividad del profesorado para que sea capaz de diseñar sus propias prácticas (por lo tanto no es un modelo a seguir o aplicar), y se ha elaborado una guía para los profesores, donde se tratan aspectos como la biografía cultural, incluyendo los patrimonios materiales a inmateriales. También se pretende interpretar el patrimonio para los jóvenes, vinculándolo con sus referentes identitarios más próximos, haciéndolo transferible a sus patrimonios personales y colectivos. Hacen referencia a una "cultura real del patrimonio cultural". El docente adopta un rol de mediador e intérprete que debe conectar los contenidos curriculares con los referentes identitarios de los alumnos y los referentes locales y colectivos. En esta guía para el profesor (realizada en los 5 idiomas de los países participantes) encontramos reflexiones y definiciones de lo que es patrimonio, referencias a la identidad europea, aprender desde y con nuestro patrimonio, pautas y líneas de actuación con y desde el patrimonio en todos los niveles educativos y ejemplos de 14 buenas prácticas educativas.

En el ámbito internacional, la Unión Europea colabora con las organizaciones internacionales y los países no miembros, con los cuales ha firmado acuerdos de cooperación o de asociación. Así, la Unión Europea está trabajando con UNESCO para preservar la herencia cultural del mundo, por ejemplo dentro del proyecto de Heritage.Net.

La protección del Patrimonio Mundial también se trata en los acuerdos concluidos con los países socios de la unión. Así, EUROMED Heritage es un programa regional que fomenta el desarrollo del patrimonio cultural en el área mediterránea europea; forma la parte del programa de cooperación con los países mediterráneos (MEDA). Euromed Heritage IV (2008-2011) crea nuevas oportunidades para que la gente comparta y se apropie simbólicamente de su extraordinario patrimonio cultural compartido y común.

EUMEDIS es una iniciativa diseñada para promover el desarrollo en los países mediterráneos de servicios digitales, tales como servicios multimedia que proporcionen la información sobre activos culturales y sitios turísticos. Los esfuerzos de proteger y de realzar patrimonio cultural también se están emprendiendo como parte de las actividades de la cooperación entre el EU y los países de África, del Caribe y del Pacífico de conformidad con el Acuerdo de Cotonou. 
Son significativos también algunos premios de la Unión Europea para la conservación y mejora del patrimonio cultural, premio Europa Nostra. En la Categoría 4 "Educación, formación y sensibilización (año 2008)", obtuvo el premio el "Centro internacional de formación en conservación del patrimonio del castillo de Bánffy en Bonţida (Rumanía): muy valorado por su planteamiento doble, consistente en la formación en materia de conservación y la conservación a través de la formación. Europa Nostra es una asociación creada en 1963 como un conjunto de organizaciones no Gubernamentales de patrimonio. En 2002, La Comisión Europea organiza la European Union Prize for Cultural Heritagel Europa Nostra Awards, con su programa en la promoción de la excelencia en la conservación del patrimonio, dentro del programa Cultura 2000.

En el ámbito específico de las actuaciones de países, destaca The Oak of Finland, una red de cooperación para la educación del Patrimonio Cultural, formada por el Consejo Nacional de Antigüedades, el Consejo Nacional finlandés de la Educación, el Ministerio de Medioambiente de Finlandia y la administración medioambiental finlandesa. La red está abierta para las escuelas, los museos, los centros ambientales y ONGs. Las operaciones comenzaron como proyecto en el periodo 1998-2004 y ahora continúan como red. Su meta principal es conseguir que los finlandeses estén muy próximos a su propio patrimonio cultural y reforzar el papel del patrimonio cultural en la educación para:

- Desarrollar las habilidades de los estudiantes a la hora de fomentar la vivencia del patrimonio cultural.

- Apoyar la cooperación entre las escuelas y los expertos del patrimonio cultural material y del ambiente cultural.

- Familiarizar tanto a los profesores como a los estudiantes con los servicios y recursos que los museos proporcionan y entenderlos como "medioambientes educativos" donde aprender.

- Producir materiales didácticos multidisciplinarios relacionados con el patrimonio cultural.

Destaca de este proyecto la potente colaboración entre diferentes agentes sociales y educativos. La red permite diseñar materiales didácticos, trabajar la educación complementaria y encontrar compañeros para la cooperación. La red incluye numerosos agentes sociales, educativos, fundaciones y asociaciones vinculadas a la educación, que desarrollan juntos métodos de educación para el patrimonio cultural.

Lo más interesante de Oak of Finland es la estructura, que abarca y complementa los niveles nacional, regional y local en las que están implicados numerosos agentes sociales: 1. Nivel nacional: Consejo Nacional finlandés de la educación, Consejo nacional de antigüedades, el Ministerio de Medioambiente. Se encargan de generar materiales de apoyo para proyectos educativos, de la enseñanza complementaria superior para los profesores en educación del patrimonio cultural, así como de realizar consultas y generar información para compartir; 2. Nivel regional: Museos regionales, museos especiales, centros ambientales, administraciones educativas. Se encargan del desarrollo de proyectos locales y de generar materiales educativos enfocados al ámbito regional; 3. Nivel local: Museos, ONGs, asociaciones y escuelas. Se ocupan de la educación local del patrimonio cultural y de generar acontecimientos inspirados en las tradiciones. 
El Gobierno Francés es, sin duda, el que más ampliamente desarrolla las líneas de educación patrimonial, con especial atención a la diversidad cultural y a los patrimonios europeos, con acciones destacadas en este sentido como La Coalición Francesa para la Diversidad Cultural, que reúne 51 organizaciones profesionales del sector cultural (cine, televisión, espectáculo en directo, edición, música, artes gráficas, artes plásticas y multimedia) y la televisión pública (ARTE y France Televisión). Defiende la facultad de los Estados para definir y aplicar sus propias políticas culturales. La finalidad de la Coalición Francesa es sensibilizar y movilizar las organizaciones profesionales culturales y las autoridades extranjeras frente a los retos planteados por la diversidad cultural. Interviene en el marco del Comité Internacional de Enlaces de Coaliciones para la Diversidad Cultural (CIL), que reúne 34 coaliciones de todo el mundo. También destacan las inversiones en Francia para la diversidad cultural como el Programa de protección de patrimonios audiovisuales y promoción de la diversidad cultural: 4 millones de euros en tres años. Conviene Destacar el papel central del patrimonio en los denominados "Estudios Artísticos y culturales" franceses, donde el patrimonio se vincula a los museos y archivos.

Efectivamente, en el ámbito francés lo patrimonial se vincula a lo artístico y cultural, y la educación del patrimonio ha de entenderse como "Educación para el patrimonio": para sensibilizar, para comprenderlo y valorarlo. La dimensión identitaria es clave, de forma que trabajar con patrimonio implica trabajar con referentes de la identidad nacional y regional, con vínculos de propiedad, pertenencia y legado.

En el ámbito anglosajón, el término "Heritage Education" se vincula mucho más a la educación del patrimonio o en el patrimonio, influidos por la disciplina de la interpretación del patrimonio, destacando los contenidos y su comprensión. Se prioriza el acceso, y que ese acceso sea de todos y para todos. Así, por ejemplo, en el ámbito de la educación especial, encontramos el programa "Heritage for all" (Patrimonio para todos), un programa de 3 años de duración en el National Trust en el Wessex Area (Wilts. Glos, Bristol, Bath, Dorset \& Somerset) que desarrolla los vínculos de la “Workers' Educational Association's (WEA's)", con personas discapacitadas de la región. La National Trust trabaja para preservar y proteger tanto edificios como el ámbito natural y la costa de Inglaterra, Gales y el Norte de Irlanda a través de la conservación, el aprendizaje y el descubrimiento. Su programa educativo comprende líneas de actuación con escolares, profesores, familias, niños, adultos. Trabaja, además, con metodologías tan diversas que abarcan desde la propia experiencia in situ, pasando por las historias de vida (orales) o un banco interactivo de la memoria. Esta iniciativa, que incide en la educación del patrimonio intercultural, permite compartir experiencias y recuerdos, se trata de la "Nations`Memorybank website" que crea lo que denominan mapas interactivos de la memoria.

\section{ESPAÑA: UN PAÍS EMERGENTE EN EDUCACIÓN PATRIMONIAL}

El volumen de patrimonio a conservar y gestionar en un país como España comprende más de 60000 Bienes de Interés Cultural (BIC) y 44 bienes incluidos en la lista de patrimonio mundial de la UNESCO, tan solo por detrás de China e Italia. Con todo, el volumen de patrimonio con el que tiene que operar un país como España es inmenso, de manera que un factor de sostenibilidad es la rentabilidad, si bien esta no tiene que ser necesariamente en términos económicos; no, al menos, de forma directa. Lo cierto es que 
otras formas de rentabilidad como la turística, social, identitaria o cultural revierten, a medio y largo plazo y con políticas adecuadas, en un beneficio económico para el conjunto de la comunidad, región o país.

Para gestionar estos bienes desde el ámbito educativo, España se ha dotado en los últimos 5 años de dos instrumentos que pretenden sistematizar, ordenar y coordinar al Estado y las Comunidades Autónomas en materia de educación patrimonial, ambos financiados por dos Ministerios del Gobierno:

$1^{\circ}$. El Plan Nacional de Educación y Patrimonio-Ministerio de Educación, Cultura y Deporte.

$2^{\circ}$. El Observatorio de Educación Patrimonial en España-Ministerio de Ciencia e Innovación.

Por su parte, la legislación en materia patrimonial apuesta por un tratamiento integral que comprende la intervención educativa, a la que concede una responsabilidad social clave en todo el proceso. Así, se pretende combinar:

la acción vigilante con el estímulo educativo, técnico y financiero, en el convencimiento de que el Patrimonio Histórico se acrecienta y se defiende mejor cuanto más lo estiman las personas que conviven con él, pero también cuantas más ayudas se establezcan para atenderlo, con las lógicas contraprestaciones hacia la sociedad cuando son los poderes públicos quienes facilitan aquéllas (Ley 16/1985, preámbulo).

De esta forma, la mentalidad de mantenimiento del bien —en su dimensión histórica, tangible, material-, va dejando paso a una conceptualización basada en la conservación y gestión integral, que comprende la educación, en torno a esos vínculos con las personas; no se trata tanto de declarar la necesidad de protección como de educar a la sociedad para que esta sea quien reclame dicha necesidad de custodia y cuidado. En este sentido, la Ley 16/1985 de Patrimonio Histórico Español hace referencia explícita, ya desde su preámbulo, al patrimonio Histórico Español como.

riqueza colectiva que contiene las expresiones más dignas de aprecio en la aportación histórica de los españoles a la cultura universal. Su valor lo proporciona la estima que, como elemento de identidad cultural, merece a la sensibilidad de los ciudadanos, porque los bienes que lo integran se han convertido en patrimoniales debido exclusivamente a la acción social que cumplen, directamente derivada del aprecio con que los mismos ciudadanos los han ido revalorizando (Ley 16/1985 de Patrimonio Histórico Español).

De este modo, se sitúa a la educación como una forma de intervención rentable para la gestión integral del patrimonio, en tanto permite que las personas contemplen y disfruten aquellas obras que son su herencia, resultado de la capacidad colectiva de un pueblo

porque en un Estado democrático estos bienes deben estar adecuadamente puestos al servicio de la colectividad en el convencimiento de que con su disfrute se facilita el acceso a la cultura y que ésta, en definitiva, es camino seguro hacia la libertad de los pueblos (Ley 16/1985). 


\subsection{EL PLAN NACIONAL DE EDUCACIÓN Y PATRIMONIO}

Casi tres décadas después de la Ley 16/1985 de Patrimonio Histórico Español, esta idea es retomada y desarrollada ampliamente en el Plan Nacional de Educación y Patrimonio (en adelante PNEyP), aprobado en junio de 2013, profundizando en este concepto de rentabilidad.

Los planes nacionales son elaborados y ejecutados desde la década de los años 80 por el Instituto del Patrimonio Cultural de España. Permiten establecer una metodología de actuación unificada sobre conjuntos de bienes culturales, programar las inversiones de acuerdo con las necesidades de conservación y coordinar la participación de las distintas instituciones que intervienen en la conservación de esos conjuntos patrimoniales.

En la estructura de estos planes, además de definirse una serie de objetivos generales y específicos, se establece lo que se denomina criterios metodológicos que, a su vez, van a dar lugar a un conjunto de programas y líneas de actuación. Precisamente, entre esos criterios metodológicos se hace referencia a la formación y especialización, la interdisciplinariedad, la flexibilidad, la participación e implicación social, la sensibilización, la calidad y la diversidad del patrimonio, de los múltiples valores que se proyectan sobre él, de los públicos y de las actuaciones que pueden llevarse a cabo a través de la educación. Junto a esos criterios se plantea uno directamente referido a la rentabilidad social, cultural e identitaria.

En el año 2010, el Instituto del Patrimonio Cultural de España, en colaboración con las Comunidades Autónomas, comenzó una exhaustiva revisión de todos estos planes con el propósito de recoger la ampliación de que ha sido objeto el concepto de patrimonio cultural —no solo porque se ha ido proyectando una visión más integral y multidisciplinar del patrimonio, sino también porque han ido surgiendo nuevas categorías-, así como la revisión de aspectos de coordinación y funcionamiento de estos planes. Uno de estos nuevos planes, de clara naturaleza transversal, es el Plan Nacional de Educación y Patrimonio, que pretende convertirse en una herramienta eficaz de coordinación entre las administraciones e instituciones gestoras de programas educativos relacionadas con la transmisión del Patrimonio Cultural (Domingo, Fontal y Ballesteros, 2013). El plan pretende lograr los siguientes objetivos:

1. Definir las bases teóricas y criterios de la educación patrimonial.

2. Fomentar la investigación en educación patrimonial.

3. Incorporar la educación patrimonial como línea de desarrollo prioritario en el marco de los planes estratégicos del Ministerio de Educación, Cultura y Deporte y de las Comunidades Autónomas.

4. Implementar la normativa educativa, al objeto de favorecer la inserción curricular de contenidos relacionados con el Patrimonio Cultural, su preservación, valoración y disfrute público.

5. Crear instrumentos de coordinación que garanticen la colaboración entre educadores y gestores del Patrimonio Cultural en materia de educación patrimonial.

6. Fomentar la elaboración de materiales educativos orientados a la transmisión de los conceptos y valores patrimoniales.

7. Integrar las líneas de actuación orientadas a la didáctica de los bienes patrimoniales en las herramientas de gestión del Patrimonio Cultural, tales como Planes Nacionales, Planes Especiales, Planes de Salvaguarda, etc. 
8. Difundir los programas y acciones educativas relacionados con el Patrimonio Cultural realizados en todo el territorio español.

9. Promocionar la cooperación española en programas y acciones internacionales de educación patrimonial, potenciando su incorporación en políticas y redes de naturaleza supranacional (Domingo, Fontal y Ballesteros, 2013).

Para concretar estos objetivos, el Plan nacional se desarrolla a través de dos programas con sus respectivas líneas de actuación:

Programa Investigación en educación patrimonial e innovación en didáctica del patrimonio. Contiene las siguientes líneas de actuación:

- La observación permanente de la educación patrimonial en España, centrada en el inventario, análisis y evaluación de programas educativos.

- El desarrollo de nuevas líneas de investigación centradas específicamente en epistemología de la educación patrimonial, en modelos educativos emergentes y en diseños y aplicaciones didácticas.

- La integración de las Tecnologías de la Información y de la Comunicación (TIC) en nuevos modelos de enseñanza-aprendizaje.

- La innovación en los recursos y herramientas para la interpretación del patrimonio.

- La investigación sobre los modelos de evaluación de los programas de educación patrimonial.

- El fomento y creación de grupos de investigación en educación patrimonial.

- La consolidación y desarrollo de la comunidad científica sobre educación patrimonial en España.

Programa Formación de educadores, gestores y otros agentes culturales e investigadores en educación patrimonial. Son líneas de actuación de este programa:

- La formación de educadores, que deben especializarse en los contenidos patrimoniales, así como en aspectos teóricos y metodológicos de la educación patrimonial.

- La formación de gestores culturales mediante el diseño y desarrollo de programas de formación, definiendo estrategias de planificación y de comunicación social, buscando la formación continua de los gestores.

- Formación de otros agentes vinculados al patrimonio como profesionales del sector turístico, profesionales de los medios de comunicación, personal de los Cuerpos y Fuerzas de Seguridad del Estado, Comunidades Autónomas y Entes Locales.

- Formación de investigadores en educación patrimonial.

- Coordinación y comunicación entre educadores, gestores de patrimonio, otros agentes culturales e investigadores. Para ellos se crearán unidades interinstitucionales y se constituirán grupos de innovación educativa.

Este plan nacional tiene una vigencia de diez años y podrá ser sometido a revisión a los cinco. Su funcionamiento será competencia de la Comisión de Seguimiento, entre cuyas funciones está el control del cumplimiento de los objetivos del plan, así como de los programas y líneas de actuación para la elaboración de los correspondientes informes anuales. 


\subsection{EL OBSERVATORIO DE EDUCACIÓN PATRIMONIAL EN ESPAÑA}

El Observatorio de Educación Patrimonial en España (OEPE) es una sucesión de dos proyectos de $\mathrm{I}+\mathrm{D}+\mathrm{i}$ financiados por el Ministerio de Economía y Competitividad, dentro de la convocatoria de Investigación fundamental no orientada, que tiene su primer desarrollo entre enero de 2010 y diciembre de 2012 (EDU2009-09679) y un segundo trienio entre enero de 2013 y diciembre de 2015 (EDU2012-37212). El equipo de investigación está compuesto por 20 investigadores procedentes de 9 áreas de conocimiento, de 7 universidades españolas y una francesa.

Desde 2010, el observatorio ha generado una base de datos (BdD OEPE) con más de 1.200 entradas, que inventaría hasta 19 tipologías educativas de programas sobre educación patrimonial diseñados e implementados en España y en el ámbito internacional, especialmente en la última década, aunque hay referencias desde 1998. Por otro lado comprende un portal web (Portal OEPE) que sirve como herramienta de difusión de la educación patrimonial, que refleja los programas inventariados más significativos en la $\mathrm{BdD}$ (3 por tipología), aportando información geolocalizada de los proyectos registrados y analizados, además de contener una sección dedicada a la investigación en educación patrimonial. A través de la conexión con Redes Sociales se genera un espacio de debate y se difunde información actualizada de los eventos científicos sobre educación y patrimonio, seminarios, investigaciones y tesis doctorales en educación patrimonial. El trabajo del observatorio, desde 2010 hasta 2015, se ordena en 6 fases paralelas y, en ocasiones, simultáneas:

Gráfico 1. Fases que ordenan las actuaciones del OEPE

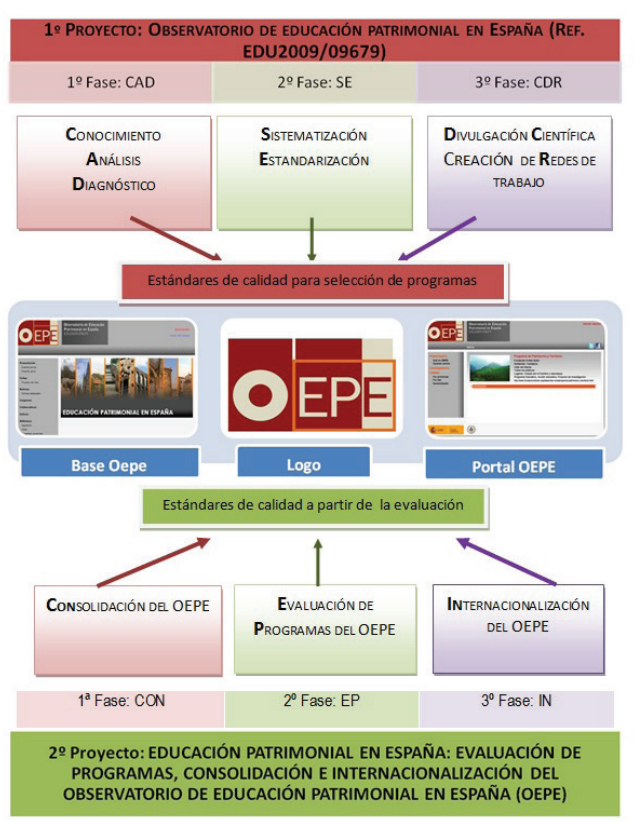

Fuente. Elaboración propia. 
Desde el OEPE entendemos que es necesario no solo encontrar programas educativos sobre patrimonio que se estén generando o se hayan generado con mayor o menor difusión y visibilidad, sino hacerlo conforme a criterios y descriptores de búsqueda claros. Todo ese volumen de propuestas educativas ha de ser convenientemente almacenado y clasificado de cara a poderlo ordenar y analizar en base a diferentes tipologías, enfoques, criterios subyacentes, etc. Ese volumen de propuestas educativas puede permitirnos medir desde la situación de la educación patrimonial de nuestro país o su evolución en la última década, hasta los modelos educativos predominantes y las ausencias determinantes. Solo así podremos mostrar lo que hacemos exhaustivamente y estaremos en disposición de colaborar con otros países, e incluso de liderar proyectos internacionales.

Así pues, a partir de 23 criterios de selección y 14 de exclusión convenientemente definidos y desarrollados, se han seleccionado más de 2000 programas para inventariar hasta diciembre de 2015, de los que ya se han introducido más de 1200 en la BASE de datos OEPE (846 españoles y 402 internacionales referidos a 43 países), clasificados en base a 19 tipologías de programa diferentes en función de su naturaleza educativa, y 18 categorías en base a su naturaleza patrimonial. Desde esta base de datos se gestiona el inventario de los programas educativos, mediante una ficha de recogida de datos que se organiza en 5 grandes apartados: (1) Identificación y localización, (2) Descripción, (3) Datos del diseño educativo, (4) Relación con otras fichas y (5) Anexo documental. En conjunto, la ficha de inventario se compone de 42 campos más un anexo documental que incluye documentos escritos, audiovisuales y links.

\subsection{DATOS RELEVANTES SOBRE LA EDUCACIÓN PATRIMONIAL}

Este volumen de programas educativos es sometido periódicamente a análisis estadístico descriptivos, análisis de contenido y, tras una selección de programas referentes, una evaluación basada en estándares.

Estos análisis nos permiten conocer datos de gran interés para comprender el estado de la educación patrimonial en el ámbito internacional. Por ejemplo, sabemos que la mayoría de los programas localizados se centran en el Patrimonio Cultural $(43,48 \%)$ y el Patrimonio Inmaterial $(15,5 \%)$, frente a categorías de baja presencia como el Patrimonio Digital $(0,35 \%)$, inscripción en cavernas y construcciones aisladas (ambas $0,46 \%$ ), obra pictórica $(0,58 \%)$ o lugares especiales $(0,81 \%)$, entre otros. Esto implica que el patrimonio cultural ha ido superando a otras visiones más historicistas. También se han notado las consecuencias del creciente interés hacia el patrimonio cultural desde 2003 (año del tratado internacional de la UNESCO sobre esta tipología) y, al tiempo, se pone de manifiesto el escaso interés por elementos vinculados a la prehistoria o, en el otro extremo, los patrimonios más contemporáneos, llamados emergentes. En cuanto a las tipologías educativas, la mayoría se refieren a programas $(19,3 \%)$, proyectos $(15,8 \%)$, recursos $(10,2 \%)$, diseños educativos $(9,9 \%)$ frente a otras con muy baja presencia como proyectos de mejora $(1,1 \%)$, lo cual indica la tendencia a una cierta "hiperactividad" de la educación patrimonial, a proyectos inmediatos en el corto plazo, sin mucha reflexión antes, durante y después de los diseños e implementaciones.

Por otra parte, es muy preocupante que solo un $65,1 \%$ de los programas inventariados en OEPE explicite objetivos; si bien es la mayoría, el estándar en este sentido es claro, en tanto todo proyecto educativo ha de exponer claramente sus objetivos didácticos. Dentro de la educación formal, la Educación Secundaria Obligatoria es objeto de más programaciones 
que las etapas de Infantil y Primaria, lo que se corresponde con una mayor presencia de los contenidos patrimoniales en el currículum en esta etapa y, por lo mismo, por la menor presencia de estos contenidos en la educación primaria e infantil respectivamente (Fontal, 2011). En el ámbito no formal encontramos una gran dispersión de destinatarios: un $13,4 \%$ dirigidos a todos los públicos y un $6,4 \%$ a niños y jóvenes, frente a otros colectivos levemente presentes como personas con discapacidad (solo un $0,6 \%$ ) o familias $(0,3 \%)$. Esto es un indicador de la tendencia a ordenar los públicos del ámbito formal a partir de los niveles y etapas educativas del ámbito formal y, además, constata una ausencia o escasez alarmante en la educación patrimonial con colectivos diversos (Marín, 2013).

Si nos fijamos en el grado de concreción educativa, hallamos muy bajo nivel de estructuración y consistencia desde parámetros propios del diseño, programación e implementación didácticos. Por ejemplo, un 44,9\% de los programas inventariados no explicitan "objetivos didácticos". De entre los objetivos que sí se explicitan, los verbos más empleados son sensibilizar, valorar, conocer y participar, entre otros. Esto nos sitúa ante objetivos preferentes que sí abarcan toda la secuencia procedimental: conocer para comprender, comprender para valorar, valorar para sensibilizar, sensibilizar para cuidar, cuidar para disfrutar y disfrutar para transmitir (Fontal, 2003).

El 81,7\% tampoco define una "justificación" en sus planteamientos. Prácticamente la mitad (54,5\%) describe los "contenidos principales" a los que atienden los programas, destacando aquellos que abordan contenidos patrimoniales (patrimonio histórico, artístico, cultural, inmaterial), así como cuestiones relativas a los valores y aspectos identitarios que se derivan de la educación patrimonial. Esto significa que la dimensión simbólico-identitaria sí está presente al menos conceptualmente en una parte significativa de programas.

Solo un $27,9 \%$ de los programas describen los "planteamientos metodológicos" en los que se sustentan, sobresaliendo las metodologías participativa, activa, integradora, práctica, con los términos que se emplean textualmente para definirla. Analizando el contenido de las propuestas, estas suelen centrarse en la transmisión de contenidos (64,2\%), en la comprensión $(52,2 \%)$, en la interpretación $(50,3 \%)$, frente a la práctica ausencia de los procesos de sensibilización $(0,7 \%)$, identización $(0,2 \%)$ o puesta en valor $(3,1 \%)$. Estos datos muestran una tendencia hacia enfoques transmisivos e interpretativos frente a enfoques procesuales, que entiendan el trabajo educativo con lo patrimonial como una sucesión de procesos que, si bien parten del conocimiento y comprensión, han de transitar por procesos relacionados con la dimensión específicamente patrimonial tales como la valorización (o proyección de mayores), la sensibilización o la identización. Estos datos demuestran, además, que si bien en la concepción del patrimonio estamos transitando de una visión más estática a otra más dinámica, como explicábamos al comienzo de este artículo, el diseño e implementación se sostienen, mayoritariamente, sobre conceptos de patrimonio superados o ya complementados. Esto no hace sino evidenciar la desconexión entre teoría y práctica, entre conceptualización sobre patrimonio y diseño didáctico.

Sin embargo, seguramente uno de los datos que más preocupa es que únicamente el $1,9 \%$ de estos programas contemple y explicite algún mecanismo de evaluación, es decir, la intención de conocer si el programa ha sido efectivo, eficaz, si ha logrado los objetivos previstos, si han aparecido otros inicialmente no previstos, cuáles son los aprendizajes y su alcance; la evaluación de estas iniciativas es el único modo de conocer la rentabilidad social, cultural e identitaria de tantísimo esfuerzo económico y recursos humanos empleados para la gestión del patrimonio cultural, su educación y difusión. 
Todos estos datos no hacen sino corroborar la necesidad de un seguimiento y medición constantes de la educación patrimonial en aras a lograr programas educativos sólidos, sustentados sobre principios psicopedagógicos, capaces de objetivar y, en consecuencia, medir las acciones en materia de educación patrimonial que se generan en nuestro país. Además, de este modo se conseguirá cambiar la inercia de la "hiperactividad" por otra más próxima al pensamiento sobre la acción pensada (Fontal, 2012), que se base en la reflexión constante y en la teorización a partir de los procesos de implementación.

Por otra parte, se ha realizado una selección por parte de expertos y basada en los estándares resultantes de los primeros 3 análisis, de 38 programas y actividades recogidos por el Observatorio (2 por tipología), y están siendo objeto de una evaluación centrada en su enfoque teórico, metas, la calidad de los diseños, la planificación de las actuaciones, el grado de cumplimiento y adecuación de la planificación (implementación), así como la calidad de los resultados e impactos generados (De Miguel, 2000). Esta evaluación basada en criterios, cimentada sobre el análisis de variables descriptivas, nos permite conocer cómo son esas propuestas, quién las diseña e implementa, si se evalúan en algún momento, si plantean objetivos claros, si la metodología docente es adecuada a los objetivos, si se diseñan propuestas para "todo tipo" de personas, si existe pensamiento y reflexión en torno a ellas, cuánto tiempo duran, quién participa en ellas o qué repercusión tienen, entre otros parámetros. Esta evaluación basada en estándares, más cuantitativa, no es incompatible con una evaluación cualitativa, que Stake prefiere denominar comprensiva o interpretativa en tanto "se desarrolla sobre el conocimiento experiencial y personal en un espacio y tiempo reales y con personas también reales" (Stake, 2006: 22). De este modo, la evaluación basada en estándares se combina con un estudio de casos que permite conocer la valoración por parte de todas las personas implicadas en el proceso educativo. Con todo, podemos determinar aquellos referentes nacionales e internacionales que pueden servir como modelo con el fin de generar nuevos diseños educativos en cada tipología. Todo ello, a su vez, genera la definición de ocho modelos diferentes de educación patrimonial, que permiten una aproximación inicial al análisis integral y el estado de la educación patrimonial en España.

\subsection{IBEROAMÉRICA COMO REFERENTE MUNDIAL EN EDUCACIÓN PATRIMONIAL}

Cuando se someten los programas inventariados en el observatorio a una evaluación basada en estándares, encontramos lo que denominamos "programas referentes", que pueden entenderse como clave en la generación de modelos de referencia internacional, cuyas propuestas son transferibles y se adecuan a los presupuestos internacionales. El $76 \%$ de estos programas se localizan en Iberoamérica y son Brasil, Chile, Argentina y Colombia los países que mayor número de aportaciones proponen, por este orden. Por ello, seleccionamos para el presente artículo una propuesta referente de cada uno de ellos.

Brasil: La casa de la memoria. Se trata de un proyecto coordinado por la educadora y artista visual Liliana Amaral (http://casadamemoria.wordpress.com/). El objetivo del proyecto es estudiar la memoria individual y colectiva de los habitantes de la Villa de Paranacabia, situada entre los 100 sitios destacados de Brasil como Patrimonio de la Humanidad por la UNESCO. La Casa de la memoria se basa en procesos de colaboración con la perspectiva de la propiedad, pertenencia y significación del patrimonio tangible e intangible, el establecimiento de vínculos entre el arte y la vida, así como la potenciación de las miradas individuales y colectivas de la ciudad imaginaria y sus representaciones. 
Chile: Estratigrafía de la memoria. En Chile nos proponen el modelo pedagógico de intervención social cuya misión es reconstruir la historia local desvelando y resignificando huellas y remanentes presentes en la comunidad, a través de un proceso educativo formal, articulado por una serie de actividades y juegos que pretenden desentrañar algunos aspectos de la historia comunitaria presentes en la tradición oral y cultura material. La propuesta se lleva a cabo en la Isla de Chiloé, en las comunidades costeras de Astilleros y Rauco, habitadas por agricultores y pescadores que han tenido que reciclarse como mano de obra para las empresas salmoneras de la zona. Su premisa es lo observado es lo que conocemos; desarrollan un trabajo de identificación de categorías que definen la relación de los niños (y comunidad) con los conchales. Cada actividad implica la transferencia de ciertos conocimientos no locales, que son parte de los procesos de intervención. En realidad se trata de la transferencia de nuevas categorías (resignificar) para agregar valor o cambiar ciertas percepciones negativas que se tienen sobre el patrimonio cultural comunitario, es decir poner en valor en el más amplio sentido de la palabra, desvelar su condición de recurso y capital cultural, resaltar sus posibles usos y su utilización como un recurso para el desarrollo de turismo cultural y de los museos locales.

Argentina: Te presto los ojos. Ofrece una visión narrada del patrimonio, a través de la observación de los alumnos de una escuela que acompañan al lector en sus relatos, descubriendo las historias de la ciudad. Se incluye un patrimonio olfativo y auditivo que permite descubrir, de la mano de los niños, su vivencia íntima con el patrimonio. Estos relatos se comparten en un blog invitando a compartir pensamientos y diálogos de otras personas o escuelas. Trabajan con una población que vive en Villas, en su mayoría provenientes de países limítrofes que solo se trasladan de sus hogares a la escuela, y allí en muchos casos termina su recorrido. La escuela se propuso salir, recorrer, conocer, siempre bajo el lema "no se ama lo que no se conoce", para amar, valorizar y cuidar el patrimonio.

Colombia: El patrimonio en el aula de clase. Se trata de un conjunto de recursos pedagógicos que favorecen la incorporación de la noción de patrimonio cultural en los planes educativos de las escuelas y colegios de Colombia. Presentada en formato "bitácora", cuenta con una guía pedagógica, cuatro cuadernos didácticos sobre la idea de nación, territorio, comunidad y memoria. Este recurso pretende acercar a los niños nuestro patrimonio arqueológico a través del juego didáctico. Para ello les convertirán en arqueólogos por un día y aprenderán a utilizar sus métodos y herramientas, así como el respeto por el objeto arqueológico. El recurso cuenta también con el programa "Vigías del patrimonio", compuesto por varios cuadernos didácticos y un formulario de inscripción para que las personas puedan participar activamente en la conservación y protección del patrimonio cultural Colombiano.

\section{ENFOQUES Y MODELOS INTERNACIONALES EN EDUCACIÓN PATRIMONIAL}

En 2011, Calbó, Juanola y Vallés (2011) proponen un modelo de educación patrimonial sobre, a través de y para el patrimonio que puede servir para todas las etapas, contextos, sociedades y formas de hacer individuales y colectivas. La conjunción de estas tres preposiciones implica la educación sensorial, perceptiva, corporal, espacial, emocional, expresiva y comunicativa, activa, comprensiva, estética, cognitiva, crítica, ambiental, multicultural, ética e inclusiva (Calbó et al., 2011). 


\subsection{ENFOQUES}

A partir de estos planteamientos y los que se aportan desde el Plan nacional de educación y patrimonio (Domingo et al., 2013), podemos diferenciar, en función de la finalidad con la que la educación conciba e incorpore, el patrimonio de:

- Educación con el patrimonio, que entiende el patrimonio como un recurso didáctico.

- Educación del patrimonio, que considera el patrimonio como un contenido y lo integra en materias curriculares, en contenidos disciplinares e incluso como parte de la colección de un museo, de un sitio o lugar patrimonial.

- Educación para el patrimonio, que se orienta a la enseñanza-aprendizaje de contenidos relacionados con el patrimonio, en tanto se entiende que la acción educativa interviene, es parte activa y decisiva en la configuración de ese patrimonio que, por tanto, está en construcción.

- Educación desde y hacia el patrimonio o educación patrimonial que, dando un paso más, parte de la propia idea de patrimonio — entendido a partir de un conjunto de procesospara determinar fines educativos que tienen que ver con la conformación de procesos de patrimonialización e identización (Gómez Redondo, 2012).

\subsection{MODELOS}

A partir de estos enfoques, definimos hasta ocho modelos diferentes de educación patrimonial, en función de las variables educativas en las que sitúen su acento y tomando como referencia las investigaciones de Calaf (2009), Cuenca y Estepa (2013), Estepa (2013), Fontal (2003), Fontal y Marín $(2011,2014)$ e Ibáñez, Vicent y Asensio (2012).

El modelo transmisivo sitúa al docente o educador del ámbito no formal como experto conocedor del patrimonio. Las estrategias didácticas se basan en la transmisión de información sobre los elementos patrimoniales, fijando la atención en los aspectos más conceptuales, especialmente relacionados con datos históricos, artísticos, geográficos o geológicos. En el modelo receptivo, si bien el docente o educador facilita el aprendizaje - y por lo tanto lo adapta a las características del receptor-, es frecuente que se pretenda engarzar los contenidos patrimoniales con el bagaje previo de los alumnos o del público, lo que suele dar cabida a las experiencias previas en relación con la recepción del patrimonio, obras de arte generalmente, por tanto patrimonio artístico.

Cuando los contenidos se centran en aspectos conceptuales y elementos patrimoniales considerados específicos, cambiantes en función del nivel educativo y del ámbito, estaríamos dentro de un modelo conceptual. En cambio, en el modelo contextual la clave es dónde tienen lugar esos procesos de enseñanza aprendizaje, casi siempre coincidentes con la recepción in situ del patrimonio. También se tienen en cuenta los elementos y factores que intervienen en dichos procesos, los niveles y capacidades del receptor, su situación y necesidades sociales, las experiencias previas con el patrimonio, así como los recursos que el entorno nos ofrece.

En el modelo investigativo la variable principal es el contenido patrimonial sobre el que se quiere avanzar en el conocimiento — pretendidamente científico- y sus relaciones con el contexto. En este modelo se desarrollan programas y proyectos educativos ex profeso, asociando lo que se quiere enseñar con el lugar en que estos contenidos son significativos, vinculándolos a la historia y transmisión del patrimonio fundamentalmente. 
El modelo procesual pone el acento en las relaciones que se establecen en los procesos de patrimonialización e identización. Es un modelo centrado en los vínculos entre el bien patrimonial y el sujeto o comunidades que son propietarios potenciales de ese patrimonio, si no reales, siempre simbólicos. Se abordan procesos como la puesta en valor, la sensibilización, la transmisión, etc.

El modelo constructivo tiene como variables didácticas centrales al sujeto que aprende y sus círculos de identidad (Fontal, 2003). Desde ahí se va diversificando la relación con los bienes patrimoniales, siempre sustentados sobre aprendizajes significativos, puesto que los contenidos tendrán que engarzarse con los conocimientos previos, sobre todo experienciales, del alumno para adquirir sentido. Por último, englobando la gran mayoría de los modelos anteriores, situaríamos el modelo relacional (Fontal, 2013). En él las relaciones entre el destinatario de los procesos de enseñanza-aprendizaje, los contenidos y el contexto son el eje de articulación. Las estrategias de enseñanza aprendizaje se adaptarán a las necesidades e intereses de los destinatarios y su relación con los contenidos en el contexto donde todo ello adquiere significatividad.

\subsection{ESTÁNDARES}

Tras el análisis de los programas internacionales inventariados, desde el OEPE se han determinado 13 estándares básicos relacionados con el diseño y la implementación de programas de educación patrimonial y 57 estándares específicos vinculados a cada una de las 19 tipologías educativas.

Así, pues, llegamos al final de una secuencia que parte de los estándares definidos para realizar análisis descriptivos que muestran un general alejamiento de estos estándares. Esto ha permitido que se expliciten los ejes que ordenan la evaluación de determinados referentes que se aproximan muy de cerca a su cumplimiento. De este modo, podemos determinar ausencias generales acompañadas de ejemplos excepcionales que sitúan nuestro foco de atención en necesidades concretas y retos que deben ser cubiertos en los próximos años. Todo ello permitirá que esas excepciones puedan ser más generales, cumpliendo un conjunto de estándares básicos:

Solidez teórico-metodológica: Los programas y sus evaluaciones han de desarrollar y avanzar en el ámbito de la metateoría y teoría sobre educación patrimonial, que parta de la propia acción reflexionada. En este sentido, las investigaciones y acciones inventariadas y analizadas, se constituyen en un sustrato sobre el que construir nuevas propuestas coherentes y ambiciosas.

Consistencia y coherencia teórico-empírica: Programas, proyectos y diseños educativos consistentes, fuertemente sustentados en una base teórica específica del ámbito de la educación patrimonial y de la didáctica del patrimonio (Calaf, 2009). Evitaremos programas débiles en su formulación y dirección teórica, así como en sus orientaciones didácticas.

Continuidad y estabilidad temporal: Programas que prevean y garanticen la continuidad en el tiempo, para evitar que se invierta en recursos económicos y humanos necesarios para la puesta en marcha de iniciativas que desaparecen o cuyo rastro se pierde, a veces sin dejar una huella clara de lo que ha sucedido con ellos. En un ámbito como el patrimonio la continuidad es elemento esencial que garantiza la transmisión generacional.

Estructuración didáctica resistente a las evaluaciones: Programas cuya estructura didáctica sea capaz de sostener cualquier investigación o evaluación que pudiera realizarse 
sobre ellos. Esto sugiere la necesidad de reforzar la formación referente a los métodos y técnicas de evaluación educativa, en aquellos educadores vinculados al patrimonio o en los docentes del ámbito formal que se ocupan del mismo, aspecto que precisamente es tenido en cuenta en el programa de formación del PNEyP (Domingo et al., 2013).

Interdisciplinariedad del patrimonio y de las didácticas que se ocupan de trabajarlo educativamente. Programas que superen la actual tendencia a la parcelación de los patrimonios por tipologías (patrimonio histórico, industrial, arqueológico, etnográfico, etc.), de los públicos a los que nos dirigimos (niños por etapas y niveles educativos, adultos, con discapacidad, etc.) y de las disciplinas desde las que los abordamos (didáctica de las CCSS, didáctica de la lengua y la literatura, didáctica del arte, etc.). Programas que sean capaces de combinar, interrelacionar e integrar los diversos tipos de patrimonio (interpatrimonios), los diferentes contextos (interterritorio), los diferentes colectivos (intergeneracionales, interculturales, intergrupos) y las diferentes disciplinas que se ocupan de su gestión, incluida las propias de la educación (interdisciplinar) (Fontal, 2013).

Internacionalización de los conceptos, de los diseños y de las implementaciones: Programas que coordinen a España con el resto del panorama internacional, aumentando la presencia de nuestro país en proyectos educativos internacionales $\mathrm{y}$, por otra parte, acercando nuestro país a las directrices y tendencias que se mueven al ritmo de las corrientes en educación patrimonial de origen internacional.

Innovación en educación patrimonial: Programas que apuesten por propuestas innovadoras, capaces de avanzar en las respuestas a las necesidades de conocimiento didáctico, ambiciosas en los objetivos que persiguen, en las estrategias, metodologías, dinámicas y acciones, que no solo han de ser creativas, sino efectivas didácticamente. Esta es precisamente otra de las líneas prioritarias del PNEyP, junto con la investigación en educación patrimonial (Domingo et al., 2013).

Aprovechamiento del potencial de las TIC: Programas que, además de considerar las TIC como un medio, un vehículo e incluso un contenido patrimonial en sí mismas (Fontal et al., 2008), aprovechen su enorme potencialidad en el ámbito educativo, relacionado con la ubicuidad de los aprendizajes informales (Ibáñez et al., 2012), e incluso proporcionando instrumentos tecnológicos cotidianos, que aunque no se usan ex profeso para la enseñanza del patrimonio, forman parte de nuestros modos de percibir el patrimonio, apropiarse de él, inventariarlo y catalogarlo, incluso.

Coordinación entre agentes educativos: Programas que contemplen la colaboración entre instituciones y ámbitos educativos para lograr el trabajo conjunto, continuo entre los ámbitos formal y no formal, y colaborativo entre los agentes educativos. También el PNEyP se hace eco de esta necesidad al situar como uno de sus objetivos "La creación de instrumentos de coordinación que garanticen la colaboración entre educadores y gestores del Patrimonio Cultural en materia de educación patrimonial" (Domingo et al., 2013: 10).

Enfoques procesuales basados en los vínculos entre bienes y personas: Propuestas que reflejen la concepción dinámica del patrimonio, basada en los vínculos, las relaciones entre bienes y personas en forma de propiedad, pertenencia, identidad, valorización y sensibilización (Fontal, 2013), superando aquellas centradas en la transmisión de contenidos, en la comprensión o en la puesta en valor preferentemente. La reflexión teórica nos lleva a teorizar el patrimonio en clave procesual (Gómez, 2011) sin olvidarnos de los procesos afectivos hacia el patrimonio, de sensibilización y concienciación ciudadana, comunitaria. Estos procesos, además de ser mencionados en la normativa educativa, y objetivados en 
las programaciones educativas, requieren esfuerzos, planificación, dedicación de recursos humanos y materiales, evaluación, reflexión y, en definitiva, tiempo y espacio de implementación educativa.

Diversificación y singularización de los educandos y públicos: Programas y adaptaciones específicas para personas con diferentes discapacidades, considerando las diferentes capacidades que tienen los individuos, siempre; sus diferentes conocimientos y experiencias, tendiendo a programaciones que singularicen, desde procesos diversificantes, estos conocimientos de todo público. Además, en tanto nos basemos en el concepto de diversidad funcional, las personas con discapacidad pueden ser referentes para trabajar con todo público. Esto no debe ser incompatible con generar materiales necesariamente específicos, para adaptar la percepción y comprensión del patrimonio a toda persona (Marín, 2013).

Ordenación desde los micro-patrimonios hasta los macro-patrimonios: Programas que se ocupen de los patrimonios locales, contextualizados en comunidades vivas, activas, con problemas y necesidades reales, conectadas con otros colectivos que pueden comenzar a diseñar propuestas conjuntas; potenciando, en definitiva, las ideas de sentido de lugar y sentido de pertenencia al lugar. Si el patrimonio es la relación entre bines y personas, en concordancia con lo que las diferentes normativas establecen, estos vínculos suceden - ya desde la infancia y con bienes próximos—, antes y de forma más sólida que con aquellos más lejanos o universales. De modo que la visión macro del patrimonio, que tiene su máxima expresión en la idea de Patrimonio Mundial, debe alcanzarse desde una visión micro, de cada persona, transitando por sus círculos de patrimonio que comienzan en lo más íntimo, personal, no compartido y que van abriéndose a otros patrimonios que pertenecen a su familia, a su círculo de amigos, a su localidad, a su país, pero, incluso, a esos nuevos patrimonios que ya nacen compartidos, inter.

Incorporación de la evaluación en la programación e implementación en educación patrimonial: Programas educativos que comprendan la evaluación en su propio diseño, como un elemento natural, no tanto concebido como un punto final sino como un punto intermedio, de continuidad, de evolución, de comprensión de los propios programas (Stake, 2006).

\section{REFERENCIAS BIBLIOGRÁFICAS}

Ballart, J. (1997). El patrimonio histórico y arqueológico: valor y uso. Barcelona: Ariel.

Ballart, J. (2012). De objeto a objeto de museo: la construcción de significados. En C. Ferrer y J. Vives-Ferrándiz (Coords.), Construcciones y usos del pasado: patrimonio arqueológico, territorio y mиseo (pp. 99-113). Valencia: Museu de Prehistória de València.

Ballart, J., \& Tresserras, J. J. (2001). Gestión del patrimonio cultural. Barcelona: Ariel.

Calaf, R. (2009). Didáctica del patrimonio. Epistemología, metodología y estudio de casos. Gijón: Trea.

Calbó, M., Juanola, R., \& Vallés, J. (2011). Visiones Interdisciplinarias en educación del patrimonio. Girona: Documenta Universitària.

Cuenca, J. M. (2002). El patrimonio en la didáctica de las ciencias sociales: análisis de concepciones, dificultades y obstáculos para su integración en la enseñanza obligatoria (Tesis doctoral). Universidad de Huelva.

Cuenca, J. M. (2003). Análisis de concepciones sobre la enseñanza del patrimonio en la educación obligatoria. Enseñanza de las ciencias sociales: revista de investigación, 2, 37-46. 
Cuenca, J.M., \& Estepa, J. (2013). La educación patrimonial: líneas de investigación y nuevas perspectivas. En J. Estepa (Coord.), La educación patrimonial en la escuela y el museo: investigación y experiencias (pp. 343-355). Huelva: UH.

De Miguel, M. (2000). La evaluación de programas sociales. Fundamentos y enfoques teóricos. Revista de Investigación Educativa, 2(18), 289-317.

Domingo, M., Fontal, O., \& Ballesteros, P. (Coords.). (2013). Plan Nacional de Educación y Patrimonio. Madrid: Ministerio de Educación, Cultura y Deporte, Secretaría de Estado de Cultura.

Estepa, J. (Coord.). (2013). La educación patrimonial en la escuela y el museo: investigación y experiencias. Hueva: $\mathrm{UH}$.

Fontal, O. (2003). La educación patrimonial: teoría y práctica en el aula, el museo e Internet. Gijón: Trea.

Fontal, O. (2011). El patrimonio en el marco curricular español. Revista de Patrimonio Cultural de España, 5, 21-44.

Fontal, O. (2012). Patrimonio y educación. Una relación por consolidar. Aula de innovación educativa, 208, 10-13.

Fontal, O. (2013). Estirando hasta dar la vuelta al concepto de patrimonio. En O. Fontal (Coord.), La educación patrimonial: del patrimonio a las personas (pp. 9-12). Gijón: Trea.

Fontal, O., Darrás, B., \& Rickenmann, R. (2008). El acceso al patrimonio cultural. Retos y Debates. Pamplona: Universidad Pública de Navarra y Cátedra Jorge Oteíza.

Fontal, O., \& Marín, S. (2011). Enfoques y modelos de educación patrimonial en programas significativos de OEPE. Educación artística: revista de investigación, 2, 91-96.

Fontal, O., \& Marín, S. (2014). La educación patrimonial en España: necesidades y expectativas para la próxima década. Revista de Patrimonio Histórico, 85, 17-23.

Gómez, C. (2011). Procesos de patrimonialización en el arte contemporáneo. Educación artística: revista de investigación, 2, 108-112.

Gómez, C. (2012). Identización: la construcción discursiva del individuo. Arte, Individuo y Sociedad, 2(23), 19-28.

Ibáñez, A., Vicent, N., \& Asensio, M. (2012). Aprendizaje informal, patrimonio y dispositivos móviles. Evaluación de una experiencia en educación secundaria. Didáctica de las Ciencias Experimentales y Sociales, 26, 3-18.

LEY 16/1985 de 25 de junio de Patrimonio Histórico Español. Publicada en «BOE» núm. 155, de $29 / 06 / 1985$.

Marín, S. (2013). Una investigación para abordar y entender nuestro patrimonio desde la perspectiva de la diversidad. Pulso: Revista de investigación, 36(2), 115-132.

Stake, R. (2006). Evaluación comprensiva y evaluación basada en estándares. Barcelona: Graó.

UNESCO. (1972). Convención sobre la protección del patrimonio mundial, cultural y natural. Autor: París.

UNESCO. (2003). Convención para la Salvaguarda del Patrimonio Cultural Inmaterial. Autor: París.

UNESCO. (2005). Convención sobre la protección y promoción de la diversidad de las expresiones culturales. Autor: París. 